\title{
Air Leak Syndromes: A Rare Case of Pneumoscrotum in a Neonate
}

\author{
Jai Prakash Soni ${ }^{a}$, Sandeep Choudharya, b, Mohan Makwana ${ }^{a}$
}

\begin{abstract}
Air leak syndromes are a group of clinically recognizable disorders produced either by alveolar rupture or due to gastro-intestinal perforation or iatrogenic, following which air escapes into the tissue in which air is not normally present. Herein we report a newborn who developed pneumothorax following resuscitation by untrained staff. Subsequently child developed subcutaneous emphysema, pneumoperitoneum and pneumoscrotum and recovered spontaneously.
\end{abstract}

Keywords: Pneumothorax; Pneumoperitoneum; Subcutaneous emphysema; Pneumoscrotum

\section{Introduction}

Air leak syndromes are a group of clinically recognizable disorders produced either by alveolar rupture or due to gastrointestinal perforation or open cranial wound or iatrogenic, following which air escapes into the tissue in which air is not normally present. All the clinical types of air leak syndrome can occur among babies with spontaneous vigorous respirations (usually among larger term babies) at birth or due to increased pressure of mechanical ventilator (PEEP, PIP) during treatment of RDS, meconium aspiration syndrome and birth asphyxia $[1,2]$ or due to vigorous cardio-pulmonary resuscitation done by untrained staff or due to gastro-intestinal ischemia and congenital or acquired obstruction [3]. Recently we came across such a neonate who was given mouth to mouth and Ambu bag resuscitation at primary healthcare center and developed: pneumothorax, pneumoperitoneum, pneumoscrotum and subcutaneous emphysema [4]. The pneumoscrotum following

\footnotetext{
Manuscript accepted for publication September 17, 2015

aDepartment of Pediatrics, Umaid Hospital for Women and Children, Dr. S. N. Medical College, Jodhpur, Rajasthan 342001, India

${ }^{b}$ Corresponding Author: Sandeep Choudhary, Umaid Hospital, Department of Pediatrics, Dr. S. N. Medical college, Jodhpur, Rajasthan 342001, India. Email: sandeepbugasara@gmail.com
}

doi: http://dx.doi.org/10.14740/ijcp224w resuscitation is a rare entity. To the best of our knowledge, only a few cases have been reported in the literature till date. Thus, because of its rarity we would like to report this case.

\section{Case Report}

A $2.75 \mathrm{~kg}$ full-term, male child was delivered at primary health care center and because of asphyxia child was first resuscitated by mouth to mouth respiration and subsequently Ambu bag resuscitation was given by an untrained staff. Subsequently the baby was given oxygen by nasal prong along with parenteral fluid. Next day parents noticed swelling over chest which gradually spread to scrotum for which the baby was referred to our medical college hospital.

The child was sick, irritable anoxic and puffy on arrival. His urine output was less than $1 \mathrm{~mL} / \mathrm{kg} / \mathrm{h}$, and the oxygen saturation was $84 \%$. The laboratory workup showed: leucocytosis with a total leucocyte count of $22,000 / \mathrm{mm}^{3}$, predominantly neutrophil (N87, L12), increased C-reactive protein $(10 \mathrm{mg} /$ $\mathrm{dL}$ ), blood urea $134 \mathrm{mg} / \mathrm{dL}$, serum creatinine $1.2 \mathrm{mg} / \mathrm{dL}$ and SGPT $89 \mathrm{IU} / \mathrm{L}$. Blood gas analysis showed hypoxia, hypercarbia and respiratory acidosis. X-ray of chest PA view and flat plate abdomen was done which revealed pneumothorax, pneumoperitoneum, pneumoscrotum and subcutaneous emphysema.

Because of respiratory distress baby was managed by inter-costal tube drainage and high flow oxygen supplementation. Broad spectrum antibiotic coverage was given, and the baby kept for the initial few days on parenteral fluids and gavage feeding. The distress gradually improved, urine output increased and renal functions normalized by seventh day. On 11 th day X-ray of chest and abdomen revealed no pneumothorax and no pneumoperitoneum, but there was evidence of subcutaneous emphysema and persistence of pneumoscrotum. Therefore, inter-costal tube was removed. The scrotum swelling gradually decreased and was minimal on day 16th of admission when the baby was discharged as he was accepting breast feeds, had no respiratory distress and was euglycemic.

\section{Discussion}

Air accumulation in the scrotum can be explained by three 
patho-physiologic mechanisms.

First is spread of air from a thoracic source (pneumothorax or pneumomediastinum). In the presence of a ball valve check mechanism or high volume air flow ventilation, there will be alveolar air trapping and alveolar over distention. Because of pressure gradient across affected alveoli and adjacent tissue space, the distended alveoli will rupture at alveolar base that overlie capillaries. The air leaks at the hilum will first cause pneumomediastinum. Thereafter air ascends up along perivascular sheath and esophagus. It will lead to emphysema neck. The mediastinum also communicates with the retroperitoneum through the sterno-costal attachment of the diaphragm. This space is continuous with the flanks and the pelvis. Thus air travels downwards through perivascular sheaths of great vessels and esophagus into retroperitoneal space: perinephric space and to the scrotum if air travels along the spermatic fascia. It will manifest as retroperitoneal emphysema. Later if air leaks into posterior peritoneal cavity it will cause pneumoperitoneum. If pneumoperitoneum dissects into processes vaginalis, it will cause pneumoscrotum.

Secondly, the pneumoperitoneum is usually caused by visceral perforation $[5,6]$. It is either caused by bowel ischemia or mechanical bowel obstruction. The air can reach the abdominal wall by diffusion or through small peritoneal defects and can accumulate in the scrotum dissecting at the fascial planes. Sometimes, air can travel to the scrotal sac through a patent processus vaginalis.

Third is infection with gas-producing organism (i.e., Fournier's gangrene) of scrotum or direct introduction of air into scrotum following scrotal trauma.

Our patient developed pneumothorax, pneumoperitoneum, pneumoscrotum and subcutaneous emphysema because of rupture of alveoli following high pressure resuscitation by inadequately trained nursing staff, causing over-distension and rupture of alveoli and air leak. Patient had a good recovery on supportive care.

\section{Take home message}

Require to strengthen the Neonatal Resuscitation Program to avoid iatrogenic complications.

\section{Conflict of Interest}

None.

\section{References}

1. Goldsmith JP, Koreithin EH. Pulmonary air leak assisted ventilation of the neonate. 4th Ed. 2003:192-193.

2. Tubel H, Huch R, Krimer W. Sudden pneumo- scrotum in a ventilated Infant. Eur J Peditr. 1999;152:689-690.

3. Coppes MJ, Roukema JA, Bax NM. Scrotal pneumatocele: a rare phenomenon. J Pediatr Surg. 1991;26(12):14281429.

4. Basu S, Kumar A, Gupta AK. Complications associated with neonatal resuscitation. Resuscitation. 2009;80(1):45 .

5. Gupta R, Sharma SB. Pneumoscrotum. Indian Pediatr. 2014;51(11):942.

6. Khan YA, Akhtar J. Pneumoscrotum: a rare presentation of gastric perforation in a neonate. APSP J Case Rep. 2010;1(2):15. 